\title{
Environmental risk factors for oesophageal cancer in Malawi: A case-control study
}

\section{YB Mlombe ${ }^{1}$, NE Rosenberg ${ }^{2,3}$, LL Wolf ${ }^{2}$, CP Dzamalala ${ }^{1}, K_{\text {Chalulu }}^{4}$, J Chisi ${ }^{1}$, NJ Shaheen ${ }^{3}$, MC Hosseinipour ${ }^{2,3}$, CG Shores ${ }^{3}$}

1. College of Medicine, University of Malawi, Blantyre, Malawi

2. UNC Project-Malawi, Lilongwe, Malawi

3. University of North Carolina, Chapel Hill, North Carolina, USA

4. Queen Elizabeth Central Hospital, Blantyre, Malawi

Correspondence to: Nora E. Rosenberg

E-mail: nora_rosenberg@unc.edu

\section{Abstract}

\section{Aim}

There is a high burden of oesophageal cancer in Malawi with dismal outcomes. It is not known whether environmental factors are associated with oesophageal cancer. Without knowing this critical information, prevention interventions are not possible. The purpose of this analysis was to explore environmental factors associated with oesophageal cancer in the Malawian context.

\section{Methods}

A hospital-based case-control study of the association between environmental risk factors and oesophageal cancer was conducted at Kamuzu Central Hospital in Lilongwe, Malawi and Queen Elizabeth Central Hospital in Blantyre, Malawi. Ninety-six persons with squamous cell carcinoma and 180 controls were enrolled and analyzed. These two groups were compared for a range of environmental risk factors, using logistic regression models. Unadjusted and adjusted odds ratios and 95\% confidence intervals (CI) were calculated.

\section{Results}

Firewood cooking, cigarette smoking, and use of white maize flour all had strong associations with squamous cell carcinoma of the oesophagus, with adjusted odds ratios of 12.6 (95\% CI: 4.2-37.7), 5.4 (95\% CI: 2.0-15.2) and 6.6 (95\% CI: 2.3-19.3), respectively.

\section{Conclusions}

Several modifiable risk factors were found to be strongly associated with squamous cell carcinoma. Research is needed to confirm these associations and then determine how to intervene on these modifiable risk factors in the Malawian context.

\section{Introduction}

Malawi, a resource-limited country in southeastern Africa, has a high burden of oesophageal cancer (OC). ${ }^{1} \mathrm{OC}$ is the second most common cancer in men and the third most common cancer in women, and age-standardized rates of OC are increasing. ${ }^{1}$ In nearby African countries, OC incidence is estimated at 76/100,000, much higher than 2.2/100,000 in the United States. ${ }^{2}$ In West Africa, OC is also rare. ${ }^{3}$

Oesophageal cancer is a devastating disease with a poor prognosis. ${ }^{4}$ In Malawi, the clinical scenario for OC patients is discouraging, ${ }^{5}$ owing to late presentation, minimal OC surgical expertise, no in-country radiotherapy, and availability of only basic chemotherapy. Prevention may therefore be the most effective tool for combating OC if modifiable risk factors can be identified. Understanding the modifiable causes of $\mathrm{OC}$ in Malawi is therefore an important public health priority.

Environmental exposures may be important risk factors for OC in Malawi. In other settings, cigarette smoking has been identified as an important modifiable risk factor for OC. ${ }^{6}$ In nearby African settings smoking pipe tobacco and consuming local beer have been associated with OC. ${ }^{7}$ In Malawi, additional possible risk factors include consuming food contaminated with mycotoxins, ${ }^{8-9}$ the source of drinking water, ${ }^{10}$ the type and source of maize flour (Zea mays, or corn), the way maize is stored and processed, and the type of cooking method used. Specifically, wood-burning with subsequent release of polycyclic aromatic hydrocarbons (PAH) has been shown to be a risk factor in other settings. ${ }^{11-13}$ On mycotoxins, our hypothesis was that m'gaiwa (whole grain maize flour), being the least processed type of local maize flour, harbours the most fumonisins and aflatoxins compared to other types of maize flour. Fumonisins and aflatoxins are types of mycotoxins produced by Fusarium and Aspergillus species of fungi, respectively, and have been associated with oesophageal cancer in other studies in the region, , ,14-15 and in Iran. ${ }^{16}$ No epidemiological studies have been conducted in Malawi to explore potential environmental OC risk factors. One study in Malawi examined the geographic distribution of oesophageal cancer and found no geographic clustering, but this study did not examine specific environmental risk factors. ${ }^{17}$

We conducted a hospital-based case-control study to explore the association between multiple environmental risk factors and OC. This had not been done before in the Malawian context. Therefore, this was a hypothesis-generating study to explore modifiable environmental factors for OC in Malawi.

\section{Materials and methods Study design}

This was a case-control study conducted at Kamuzu Central Hospital $(\mathrm{KCH})$ and Queen Elizabeth Central Hospital $(\mathrm{QECH})$, the two tertiary teaching hospitals in Malawi. This study was designed to compare a range of environmental exposures within the Malawi context. One hundred and thirtythree cases and 180 controls were enrolled from January 2011 to February 2013. Healthy controls were selected from both the $\mathrm{KCH}$ and $\mathrm{QECH}$ catchment areas. The study received ethical approval from the Malawi National Health Sciences Research Committee (NHSRC) and the University of North Carolina Internal Review Board (IRB).

\section{Study population}

Adult patients with suspected oesophageal cancer were identified in hospital wards and clinics by the study team, surgeons, and staff in the general surgery departments. Potential study participants were sent to cancer clinics or the endoscopy suites for eligibility evaluation and recruitment. Inclusion criteria for cases were: being 18 years of age or older; suspicion of OC based on clinical (odynophagia, dysphagia, weight loss and/or haematemesis), radiologic (barium swallow), or endoscopic (mass seen on flexible oesophageal endoscopy) means; and providing written informed consent. Potential cases had histological confirmation of OC after undergoing resection or biopsy, or had already received recent endoscopy with biopsy. Patients were excluded if the health care provider deemed it was unsafe to perform biopsy. Unmatched healthy controls were recruited from among staff and visitors at $\mathrm{KCH}$ and QECH who were 18 years or older with no history of cancer. These persons came from the same catchment areas as cases, though not in a 1:1 ratio.

http://dx.doi.org/10.4314/mmj.v27i3.3 
Individuals unable to give a clear past medical history were excluded as controls.

\section{Clinical and laboratory procedures}

Written informed consent was obtained, either in Chichewa or English, based on the participant's language competence. Trained personnel provided discussions of the consent in the participant's preferred language and answered participants' questions. If the participant was illiterate, the form was read to him or her in the presence of a witness. Participants consented with a thumbprint or signature, depending on literacy.

Thereafter, a study questionnaire was administered to obtain demographic details and known and possible risk factors for OC.

Histopathology analysis with haematoxylin and eosin staining of biopsy tissue was conducted on all cases. Oesophageal biopsy specimens were obtained by general surgeons specializing in endoscopy or by trained endoscopists using endoscopic biopsy forceps. The specimens were labelled, as per standard pathology protocol, with the patient name, hospital medical record number and, if known, date of birth; and accompanied by a laboratory request form. Specimens were transported in formalin and those obtained at $\mathrm{KCH}$ were processed at the $\mathrm{KCH}$ Pathology Laboratory. Specimens obtained at QECH were processed at the College of Medicine Pathology Laboratory in Blantyre. Haematoxylin and eosin slides were read by board-certified pathologists at $\mathrm{KCH}$ and the College of Medicine.

HIV status was determined through evaluation of health passports and hospital files. For those participants with unknown or undocumented status, rapid HIV testing was conducted. HIV test results were returned to participants on the same day.

\section{Data management and analysis}

Study information was entered into password-protected databases. Information for all cases was entered into a MySQL database run by Apache web server. Information for controls was stored in a study-specific password-protected Microsoft Access database. For data analysis, de-identified data sets from both cases and controls were converted into SPSS version 15.0 and concatenated into a single dataset. We restricted all analyses to squamous cell carcinoma (SCC), as the different types of OC may have different aetiologies and risk factors, and SCC was the only one we had sufficient power to explore. The analysis was further restricted to cases under 64 years of age $(\mathrm{N}=96,78 \%$ ) because there were no controls 65 years or older, and thus no meaningful comparison population.

We explored several possible environmental risk factors: cigarette smoking, alcohol intake, cooking method, water source, and maize type and source. Cigarette smoking and alcohol use were dichotomized (never versus ever). Cooking method was categorized as charcoal, electricity, wood, or combination. Water sources were labelled as either treated (tap) water or untreated (e.g., river, borehole) water. There were several variables related to maize, the staple food in Malawi. We assessed type of maize, maize storage (sacks inside the house or in a granary), source of maize (selfgrown or bought), and type of maize flour used (m'gaiwa, gramil, white, or combination). M'gaiwa is milled whole grain. Gramil is de-hulled, then milled. White maize flour is dehulled, soaked, washed, dried and then milled. White maize flour supposedly contains the fewest contaminants of the three types of maize flour.

Demographic variables included age, gender, and current occupation status. From participants' current occupation statuses, we derived their socioeconomic statuses, classified as "low-income", "medium-income", and "high-income". Low-income occupation groups included subsistence farmers and unemployed participants; medium-income occupation groups included manual workers (both skilled and unskilled) and domestic workers; and high-income occupation groups included professional, technical, managerial, clerical, sales, and services jobs.

Descriptive statistics included frequencies and percentages for categorical variables, and means and standard deviations for continuous variables. The distributions of these variables between cases and controls were compared using Chisquared tests (categorical variables) and t-tests (continuous variables).

Unconditional logistic regression was used to estimate odds ratios (OR) and 95\% confidence intervals of each OC risk factor in unadjusted and adjusted analyses. Adjusted analyses were conducted to control for potential confounders. All models were adjusted for age, gender, and socioeconomic status. Some models were adjusted for other possible confounders, including cigarette smoke, wood burning, and source of maize.

\section{Results}

Eleven patients $(7.6 \%)$ declined consent. Most of the OC cases ( $\mathrm{n}=123,92 \%$ ) were SCCs. Other histopathological types of OC included adenocarcinoma (2\%), small cell carcinoma $(2 \%)$, non-specific malignancy $(2 \%)$, anaplastic carcinoma (1\%) and non-Hodgkin's lymphoma (1\%). There were 27 cases $>65$ years of age who were also excluded. We therefore included 96 SCC cases and 180 controls for analysis (Table 1).

A higher proportion of cases $(66 \%)$ than controls $(39 \%)$ were male $(\mathrm{p}<0.001)$ (Table 1$)$. OC cases were older, with a mean age of 47.5 (SD 10.3) years, compared to 36.4 (SD 10.3) for controls. The majority of cases $(70 \%)$ and half of the controls $(50 \%)$ were selected from $\mathrm{KCH}$. The majority $(64 \%)$ of cases were in the lowest socioeconomic stratum, whereas the majority $(70 \%)$ of controls were in the middle socioeconomic stratum $(\mathrm{p}<0.001)$. HIV prevalence was similar in the two groups $(20 \%$ in cases, $18 \%$ in controls, $\mathrm{p}$ $=0.5)$.

Among the oesophageal SCC cases, $40 \%$ had a cigarette smoking history, compared to $6 \%$ of controls. In unadjusted analysis, the odds of developing oesophageal SCC was 11.2 times higher among smokers than among non-smokers, and in adjusted analysis it was 5.4 times higher (Table 2). The adjusted model controlled for age, gender, socioeconomic status, and cooking methods.

Alcohol consumption was also more common in cases $(36 \%)$ than among controls (20\%). In unadjusted analysis, alcohol consumption was associated with developing oesophageal SCC $(\mathrm{OR}=2.2)$. However, when controlling for age, gender, socioeconomic status, cooking methods, and cigarette smoking, it was no longer associated with oesophageal SCC $(\mathrm{OR}=1.0)$, suggesting that the unadjusted relationship was driven by confounding factors.

The distribution of cooking methods differed considerably 
Table 1: Characteristics of cases and controls

\begin{tabular}{|c|c|c|c|c|}
\hline Category & Parameter & Cases $(\mathrm{n}=96)^{\ddagger}$ & \begin{tabular}{|l|l|}
$\begin{array}{l}\text { Controls } \\
(\mathrm{n}=180)^{\ddagger}\end{array}$ \\
\end{tabular} & $\mathrm{p}$-value \\
\hline \multicolumn{5}{|l|}{ Age (yrs) } \\
\hline & $18-34$ & $10(10.4 \%)$ & $87(48.3 \%)$ & \\
\hline & $35-44$ & $29(30.2 \%)$ & $59(32.8 \%)$ & \\
\hline & $45-54$ & $34(35.4 \%)$ & $22(12.2 \%)$ & \\
\hline & $55-64$ & $23(24.0 \%)$ & $12(6.7 \%)$ & $<0.001$ \\
\hline \multicolumn{5}{|l|}{ Sex } \\
\hline & Male & $63(65.6 \%)$ & \begin{tabular}{|l|l|}
$70(38.9 \%)$ \\
\end{tabular} & \\
\hline & Female & $33(34.4 \%)$ & $110(61.1 \%)$ & $<0.001$ \\
\hline \multicolumn{5}{|l|}{ Study site } \\
\hline & $\mathrm{KCH}$ & $67(69.8 \%)$ & $90(50.0 \%)$ & \\
\hline & QECH & $29(30.29 \%)$ & $90(50.0 \%)$ & 0.002 \\
\hline \multicolumn{5}{|c|}{ HIV status } \\
\hline & Non-reactive & $65(79.3 \%)$ & $145(82.4 \%)$ & \\
\hline & Reactive & $17(20.7 \%)$ & $31(17.6 \%)$ & 0.5 \\
\hline \multicolumn{5}{|c|}{ Economic status } \\
\hline & Low income & $58(64.4 \%)$ & $29(16.3 \%)$ & \\
\hline & Medium income & $24(26.7 \%)$ & $124(69.7 \%)$ & \\
\hline & High income & $8(8.9 \%)$ & $25(14.0 \%)$ & $<0.001$ \\
\hline
\end{tabular}

Table 2: Environmental risk factors, food habits, and oesophageal cancer

\begin{tabular}{|c|c|c|c|c|}
\hline & $\begin{array}{l}\text { Cases } \\
\left(\mathrm{n}=96^{\ddagger}\right)\end{array}$ & \begin{tabular}{|l|} 
Controls \\
$\left(\mathrm{n}=180^{\ddagger}\right)$
\end{tabular} & $\begin{array}{l}\text { Unadjusted OR } \\
(95 \% \mathrm{CI})\end{array}$ & \begin{tabular}{|l} 
Adjusted OR $\dagger$ \\
$(95 \% \mathrm{CI})$
\end{tabular} \\
\hline \multicolumn{5}{|l|}{ Cooking method: } \\
\hline Charcoal & $10(10.6 \%)$ & $65(36.1 \%)$ & Reference & Reference \\
\hline Combination & $16(17.0 \%)$ & $70(38.9 \%)$ & $1.5(0.6-3.5)$ & $1.0(0.4-2.7)$ \\
\hline Electricity & $2(2.1 \%)$ & $32(17.8 \%)$ & $0.4(0.1-2.0)$ & $0.4(0.1-2.3)$ \\
\hline Wood & $66(70.2 \%)$ & $13(7.2 \%)$ & $33.0(13.5-80.6)$ & $12.6(4.2-37.7)$ \\
\hline \multicolumn{5}{|c|}{ Cigarette smoking: } \\
\hline Never & $56(60.2 \%)$ & $170(94.4 \%)$ & Reference & Reference \\
\hline Ever & $37(39.8 \%)$ & $10(5.6 \%)$ & $11.2(5.2-24.0)$ & $5.4(2.0-15.2)$ \\
\hline \multicolumn{5}{|l|}{ Alcohol: } \\
\hline Never & $60(64.5 \%)$ & $144(80.0 \%)$ & Reference & Reference \\
\hline Ever & $33(35.5 \%)$ & $36(20.0 \%)$ & $2.2(1.3-3.9)$ & $1.0(0.3-2.9)$ \\
\hline \multicolumn{5}{|l|}{ Maize storage: } \\
\hline Sacks in the house & $62(68.1 \%)$ & $121(67.2 \%)$ & Reference & Reference \\
\hline Granary & $29(31.9 \%)$ & $59(32.8 \%)$ & $1.0(0.6-1.6)$ & $0.8(0.3-2.2)$ \\
\hline \multicolumn{5}{|l|}{ Source of maize: } \\
\hline Buy & $19(20.7 \%)$ & $124(68.9 \%)$ & Reference & Reference \\
\hline Grown own & $73(79.3 \%)$ & $56(31.1 \%)$ & $8.5(4.7-15.4)$ & $3.1(1.3-7.5)$ \\
\hline \multicolumn{5}{|c|}{ Type of maize flour used: } \\
\hline Gramil & $17(18.7 \%)$ & $100(55.6 \%)$ & Reference & Reference \\
\hline M'gaiwa & $13(14.3 \%)$ & $35(19.4 \%)$ & $2.2(1.0-10.1)$ & \begin{tabular}{|l|l|}
$1.5(0.4-5.5)$ \\
\end{tabular} \\
\hline White & $60(65.9 \%)$ & $40(22.2 \%)$ & $8.8(4.6-17.0)$ & $6.6(2.3-19.3)$ \\
\hline Combination & $1(1.1 \%)$ & $5(2.8 \%)$ & $1.2(0.1-10.7)$ & $0.5(0.0-7.3)$ \\
\hline \multicolumn{5}{|c|}{ Water source: } \\
\hline Treated (tap) water & $27(28.7 \%)$ & $103(57.2 \%)$ & Reference & Reference \\
\hline $\begin{array}{l}\text { Untreated (e.g., } \\
\text { river or borehole) } \\
\text { water }\end{array}$ & $67(71.3 \%)$ & $77(42.8 \%)$ & $3.3(1.9-5.7)$ & $0.8(0.3-1.9)$ \\
\hline
\end{tabular}

among cases and controls. The majority (70\%) of cases resided in households where firewood was used, compared to only $7.2 \%$ of the controls (Table 2 ). The majority of controls used either charcoal cooking $(36 \%)$ or a mixture of cooking methods (39\%). In unadjusted analysis, the odds of oesophageal cancer was 33.0 times higher among those who used firewood compared to those who used charcoal. In adjusted analysis, the odds of oesophageal cancer was 12.6 times higher among those who used firewood compared to those who used charcoal after controlling for age, gender, socioeconomic status, and cigarette smoking. This trend was stronger among women $(\mathrm{OR}=85.3)$ than men $(\mathrm{OR}=6.4)$.

Approximately two-thirds of cases and controls reported maize storage in the house as opposed to a granary. Most cases $(79 \%)$ grew their own maize, compared to $31 \%$ of controls. Additionally, most cases $(66 \%)$ used white maize, whereas most controls $(56 \%)$ used gramil. Type of maize storage was not associated with oesophageal SCC in unadjusted $(\mathrm{OR}=1.0)$ or adjusted $(\mathrm{OR}=0.8)$ analysis. Growing one's own maize was moderately associated with increased odds of oesophageal cancer (adjusted OR = 3.1). Using white maize flour was strongly associated with oesophageal cancer (adjusted $\mathrm{OR}=6.6$ ) after controlling for age, gender, socioeconomic status, cooking methods, smoking, and source of maize.

Use of untreated water was more common among cases $(71 \%)$ than among controls (43\%). In unadjusted analysis, water source was associated with developing oesophageal SCC $(\mathrm{OR}=3.3)$. However, in adjusted analysis controlling for age, gender, socioeconomic status, cooking methods, and smoking, it was no longer associated with oesophageal SCC $(\mathrm{OR}=0.8)$, suggesting that the unadjusted relationship was driven by confounding factors.

\section{Discussion}

Our findings suggest that wood cooking, cigarette smoking, and consumption of white maize flour were all associated with oesophageal SCC in our study population, while alcohol use, use of untreated water, and other environmental factors were not. These findings point to several modifiable factors that may contribute to the high incidence of SCC in Malawi. The finding that wood cooking was strongly associated with oesophageal SCC offers a novel explanation for the high incidence of $\mathrm{OC}$ in Malawi. Wood burning is the predominant source of energy in Malawian homes, with $85 \%$ of households reporting the use of wood as fuel. ${ }^{18}$ In rural areas, the percentage of households using wood as fuel for cooking is considerably higher than in urban areas $(94 \%$ versus $37 \%$ ). ${ }^{18}$ Wood burning was found to be more strongly associated with OC than charcoal burning in our study. These are both considered dirty fuels. ${ }^{19}$ However, because wood burning uses a naked flame to heat items, whereas charcoal uses glowing embers, wood burning produces more particulate matter, ${ }^{20-21}$ which contains products of incomplete combustion, such as PAH. A study in Sierra Leone found a higher prevalence of acute respiratory infections among children in homes with wood stoves, compared with homes with charcoal stoves. ${ }^{22}$ We could not compare the use of dirty fuels with the use of cleaner fuels, like electricity, because there were not enough electricity users in our study. But it is interesting to note that a study done in Mozambique, one of Malawi's neighbours, suggested that use of charcoal did not pose greater health risk than use of modern fuels (electricity and gas)..$^{21}$

Wood burning has been linked to oesophageal SCC in South Africa, ${ }^{23}$ Iran, ${ }^{11}$ and Brazil. ${ }^{24}$ Use of wood-burning stoves typically occurs inside the home, where ventilation is poor, contributing to indoor air pollution. In Malawi, 68\% of households cook indoors, especially in rural areas, where the majority of Malawians live. ${ }^{18}$ Malawian women tend to do most of the cooking, and we observed that firewood cooking was more strongly associated with SCC among women than men in a gender-stratified analysis. Indoor air pollution has also been associated with head and neck cancers, including $\mathrm{OC}$, in high-risk areas of central and eastern Europe. ${ }^{25}$ Head and neck cancers are prevalent in Malawi and are the fourth leading cause of cancer.

It has been postulated that high exposure to $\mathrm{PAH}$ may contribute to increased risk of OC. ${ }^{11}$ Homes with woodburning stoves tend to have higher levels of PAH than non-wood-burning homes. ${ }^{12}$ Cooking by direct heat may also be another mechanism by which wood burning could contribute to oesophageal SCC. Broiling, barbequing, and high-temperature cooking have shown association with oesophageal SCC. ${ }^{26-28}$ We did not study these aspects of cooking as risk factors, but we postulate that these could promote ingestion of $\mathrm{PAH}$ and thus could be contributory mechanisms for the association between wood cooking and oesophageal SCC.

Smoking is a well-described risk factor for oesophageal SCC, including in southern Africa. ${ }^{29}$ To our knowledge, this is the 
first time that smoking has been associated with SCC of the oesophagus in Malawi. However, $60 \%$ of the cases had never smoked, indicating that other risk factors must also play a significant role. Wood-burning stoves and cigarette smoking are both potential types of indoor air pollution. These types of indoor air pollution have also been linked to lung cancer and chronic obstructive pulmonary disease. These two diseases, together, were the third leading cause of death worldwide in 2011. ${ }^{30}$ Strategies for addressing indoor air pollution would not only have potential impacts on the burden of oesophageal cancer, but also these other public health challenges. Intervention research to assess how to reduce exposure to indoor air pollution is therefore a public health imperative.

Maize is the staple food for Malawi; it is mainly consumed as flour and can be contaminated by mycotoxins at various stages of its production and storage. We found that growing one's own maize was moderately associated with oesophageal cancer, perhaps resulting from contamination during the growing process. In contrast, our finding that white maize flour has a stronger association with SCC of the oesophagus than m'gaiwa is counterintuitive. The production of white maize flour has less mycotoxin contamination than gramil or m'gaiwa. ${ }^{31} \mathrm{We}$ expected m'gaiwa to have the strongest association with SCC. A possible explanation for the association of white maize flour with oesophageal SCC is lower fiber content in the white maize flour compared to the other less-refined forms of maize flour, such as m'gaiwa. The intake of fiber from whole grains has been associated with reduced risk for cancers of the aerodigestive tract. ${ }^{32}$ Another possibility is an unidentified toxin that is concentrated in white maize flour.

Control selection was imperfect. An important limitation of our study is that the majority of our controls were younger females of higher socioeconomic status than our cases, who were mainly poorer, older, male farmers. Additionally, because of the lack of older control subjects, we excluded the older oesophageal SCC patients. This was largely a result of the control selection process. Many of the controls were hospital staff or were recruited personally by hospital staff through convenience methods, because of time and resource constraints. We attempted to control for these factors through adjustment for age, gender, and socioeconomic status. However, our estimates may reflect bias from residual confounding.

Our study has several notable strengths. One strength is that all cases were histologically confirmed-a true challenge in our setting. Another important strength is that we had large numbers of cases and controls. Thirdly, we involved two hospitals whose combined catchment areas cover more than enough of Malawi's population to consider the study sample as representative for the country. Finally, it is desirable that controls reflect the population from which the cases are drawn, and our controls were a reasonable match for the population of Malawi, which is young with more women than men.

The risk factors for oesophageal SCC are likely multifactorial, including environmental exposures. Further research confirming these environmental risk factors, as well as the genetic-environment interactions, will be critical to the understanding of these relationships.

Our study suggests that wood-based cooking, smoking, growing one's own maize, and white maize flour are potential risk factors for oesophageal cancer in Malawi. Research with better control of confounding to confirm associations and then, ultimately, to determine how to intervene on these modifiable risk factors (e.g., with alternative cooking methods), in the Malawian context, is needed.

\section{Acknowledgements}

We would like to acknowledge Anstead Kankwatira and other staff at $\mathrm{KCH}$ and QECH for performing endoscopies and other contributions to the study. Yohannie Mlombe was supported by the Fogarty International Clinical Research Scholars \& Fellows Program of Vanderbilt University. Yohannie Mlombe and Nora E. Rosenberg were supported by R25-TW009340 (UNC Hopkins Morehouse Tulane Fogarty Global Health Fellows Program). Nora E. Rosenberg was also supported by the Center for AIDS Research 5P30AI50410. Lindsey Wolf was supported by the Doris Duke Charitable Foundation.

\section{References}

1. Msyamboza KP, Dzamalala C, Mdokwe C, Kamiza S, Lemerani M, Dzowela T and Kathyola D. Burden of cancer in Malawi; common types, incidence and trends: Nationalpopulation-based cancer registry. BMC Research Notes 2012;5:149-157.

2. Lambert R, Hainaut P. Esophageal cancer: cases and causes (part I). Endoscopy 2007;39(6):550-555.

3. Olokoba AB, Obateru OA. Oesophageal carcinoma--a report of two cases and review of literature. Nig Q J Hosp Med. 2009;19(4):186-9.

4. Kachala R. Systematic review: epidemiology of oesophageal cancer in Sub-Saharan Africa. Malawi Med J. 2010;22(3):65-70.

5. Thumbs A, Borgstein E. Commentary: managing oesophageal cancer in a resource poor setting-a Malawian example. BMJ 2010;341:c6723.

6. Pacella-Norman R, Urban MI, Sitas F, Carrara H, Sur R, Hale M, Ruff P, Patel M, Newton R, Bull D, Beral V. Risk factors for oesophageal, lung, oral and laryngeal cancers in African South Africans. Br J Cancer 2002;86(11):1751-1756.

7. Segal I, Reinach SG, de Beer M. Factors associated with oesophageal cancer in Soweto, South Africa. Br J Cancer. 1988;58(5): 681-686.

8.Williams JH, Grubb JA, Davis JW, Wang JS, Jolly PE, Ankrah NA, El lis WO, Afriyie-Gyawu E, Johnson NM, Robinson AG, Phillips TD: HIV and hepatocellular and esophageal carcinomas related to consumption of mycotoxin-prone foods in sub-Saharan Africa. Am J Clin Nutr 2010; 92(1):154-160.

9. Wild CP, Gong YY. Mycotoxins and human disease: a largely ignored global health issue. Carcinogenesis 2010;31(1):71-82.

10. Keshavarzi B, Moore F, Najmeddin A, Rahmani F, Malekzadeh A.Quality of drinking water and high incidence rate of esophageal cancer in Golestan province of Iran: a probable link. Environ Geochem Health 2012;34(1):15-26. doi: 10.1007/s10653-011-9377-3.

11. Kamangar F, Strickland PT, Pourshams A, Malekzadeh R, Boffetta P, Roth MJ, Abnet CC, Saadatian-Elahi M, Rakhshani N, Brennan P, Etemadi A, Dawsey SM. High Exposure to Polycyclic Aromatic Hydrocarbons May Contribute to High Risk of Esophageal Cancer in Northeastern Iran. Anticancer Res. 2005;25 (1B): 425-428.

12. Gustafson P, Östman C, Sällsten G. Indoor Levels of Polycyclic Aromatic Hydrocarbons in Homes with or without Wood Burning for Heating. Environ. Sci. Technol. 2008;42 (14):5074-5080.

13. Islami F, Boffetta P, van Schooten FJ, Strickland P, Phillips DH, Pourshams A, Malekshah AFT, Godschalk R, Jafari E, Etemadi A, Abubaker S, Kamangar F et al. Exposure to Polycyclic Aromatic Hydrocarbons Among Never Smokers in Golestan Province, Iran, an Area of High Incidence of Esophageal Cancer - a Cross-Sectional Study with Repeated Measurement of Urinary 1-OHPG in Two Seasons. Front Oncol. 2012; 2: 14. 
14. Rheeder JP, Marasas WFO, Thiel PG, Sydenham EW, Shephard GS, van Schalkwyk DJ. Fusarium moniliforme and fumonisins in maize in relation to human esophageal cancer in Transkei. Phytopathology 1992;82:353-357.

15. Marasas WFO, Jaskiewicz K, Venter FS, van Schalkwyk DJ. Fusarium moniliforme contamination of maize in esophageal cancer areas in Transkei. S Afr Med J. 1988;74:110-114.

16. Ghasemi-Kebria F, Joshaghani H, Taheri NS, Semnani S, Aarabi M, Salamat F, Roshandel G. Aflatoxin contamination of wheat flour and the risk of esophageal cancer in a high risk area in Iran. Cancer Epidemiol. 2013;37(3):290-3.

17. Mlombe Y, Dzamalala C, Chisi J, Othieno-Abinya N: Oesophageal cancer and Kaposi's sarcoma in Malawi: a comparative analysis. Malawi Med J 2009;21(2):66-68

18. National Statistical Office (NSO) and ICF Macro. 2011. Malawi Demographic and Health Survey 2010. Zomba, Malawi, and Calverton, Maryland, USA: NSO and ICF Macro.

19. WHO. Fuel for Life: Household Energy and Health; 2006. Available online: http://www.who.int/indoorair/publications/fuelforlife.pdf (Accessed on 16 November 2013).

20. Ezzati M, Mbinda MB, Kammen MD. Comparison of emissions and residential exposure from traditional and improved cookstoves in Kenya. Envir. Sci. Technol. 2000;34:578-583.

21. Ellegard A. Cooking fuel smoke and respiratory symptoms among women in low income areas in Maputo. Envir. Health Perspect. 1996;104: 980-985.

22. Taylor1 ET, Nakai S. Prevalence of Acute Respiratory Infections in Women and Children in Western Sierra Leone due to Smoke from Wood and Charcoal Stoves. Int J Environ Res Public Health. 2012; 9(6): 2252-2265.

23. Dandara C, Ballo R, Parker MI. CYP3A5 genotypes and risk of oesophageal cancer in two South African populations. Cancer Letters. 2005;225(2):275-282.

24. Mota OM, Curado MP, Oliveira JC, Martins E, Cardoso DMM. Risk factors for esophageal cancer in a low-incidence area of Brazil. Sao Paulo Med. J. 2013;131(1). Available at http://www.scielo.br/scielo. php?pid=S1516-31802013000100027\&script $=$ sci_arttext (Accessed 23 August, 2013).
25. Sapkota A, Zaridze D, Szeszenia-Dabrowska N, Mates D, Fabiánová E, Rudnai P, Janout V, Holcatova I, Brennan P, Boffetta P, Hashibe M. Indoor air pollution from solid fuels and risk of upper aerodigestive tract cancers in central and eastern Europe. Environ Res. 2013;120:905 .

26. De Stefani E, Ronco A, Mendilaharsu M, Deneo-Pellegrini H. Casecontrol study on the role of heterocyclic amines in the etiology of upper aerodigestive cancers in Uruguay. Nutr Cancer. 1998;32(1):43-8.

27. Hakamia R, Etemadiab A, Kamangarac F, Pourshamsa A, Mohtadiniad J, Firooziae MS, Birkettf N, Boffettagh P, Dawseyb SM, Malekzadeha R. Cooking Methods and Esophageal Squamous Cell Carcinoma in High-Risk Areas of Iran. Nutrition and Cancer. DOI: 10.1080/01635581.2013.779384.

28. Jessri M, Rashidkhani B, Hajizadeh B, Jessri M, Gotay C. Macronutrients, vitamins and minerals intake and risk of esophageal squamous cell carcinoma: a case-control study in Iran. Nutr J. 2011;10:137

29. Bradshaw E, Schonland M. Smoking, Drinking and Oesophageal Cancer in African Males of Johannesburg, South Africa. Br J Cancer. 1974;30(2):157-163.

30. The top ten causes of death. World Health Organization (WHO) fact sheet number 310. Available at: http://www.who.int/mediacentre/ factsheets/fs310/en/index.html (Accessed 8 November, 2013).

31. Matumba L, Monjerezi M, Chirwa E, Lakudzala D, Mumba P. Natural occurrence of AFB1 in maize and effect of traditional maize flour production on AFB1 reduction in Malawi. African Journal of Food Science. 2009;3(12):413-425.

32. Kasum CM, Jacobs DR Jr, Nicodemus K, Folsom AR. Dietary risk factors for upper aerodigestive tract cancers. Int $\mathrm{J}$ Cancer. 2002;99(2):267-72. 